\title{
Rezension: Abegg-Vaterlaus, Die Patentverletzung durch additive Fertigung (3D-Druck)
}

\author{
Mark Schweizer *
}

Lukas Abegg-Vaterlaus nimmt die technologische Entwicklung additiver Fertigungsmethoden zum Anlass, die Schutzwirkung von Patenten informationstheoretisch zu analysieren. Er kommt zum Schluss, dass eine Fertigungsdatei, die mit einer geeigneten Fertigungsanlage ohne weiteren menschlichen Input einen patentgeschützten Gegenstand erzeugen kann, selbst ein patentiertes Erzeugnis ist und die Erstellung der Fertigungsdatei daher auch ohne körperliche Umsetzung ausschliesslich dem Patentinhaber vorbehalten ist.

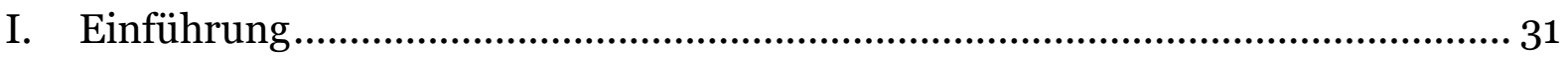

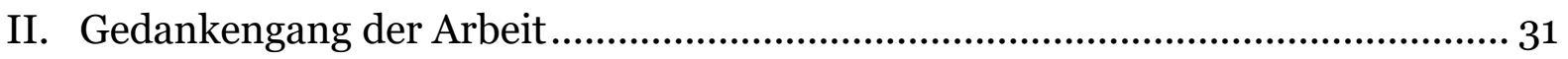

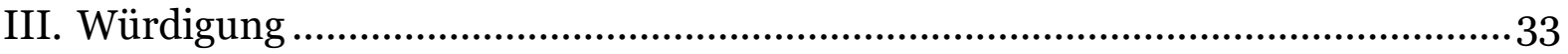

Zitiervorschlag: Mark Schweizer, Rezension: Abegg-Vaterlaus, Die Patentverletzung durch additive Fertigung (3D-Druck), in: sui-generis 2020, S. 30

URL: $\quad$ sui-generis.ch/118

DOI: $\quad$ https://doi.org/10.21257/sg.118

* PD Dr. iur. Mark Schweizer, LL.M. (Ann Arbor), Präsident Bundespatentgericht, St. Gallen (mark.schweizer@gmail.com). 


\section{Einführung}

1 Neue Technologien bieten Anlass, überlieferte rechtliche Gewissheiten zu hinterfragen. Lukas Abegg-Vaterlaus motiviert die Entwicklung additiver Fertigungsmethoden in seiner von Herbert Zech betreuten Dissertation dazu, zu untersuchen, ob die Herstellung eines patentierten Gegenstandes notwendigerweise seine körperliche Erzeugung umfassen muss, oder ob sich das patentierte Erzeugnis nicht auch in einer Fertigungsdatei manifestieren kann, die alle zur körperlichen Herstellung des patentierten Gegenstandes notwendigen Informationen umfasst. Abegg-Vaterlaus betrachtet das Patentrecht dabei durch die Linse der Informationstheorie, wie sie von Zech für das Recht fruchtbar gemacht wurde.

\section{Gedankengang der Arbeit}

2 Einleitend werden die Grundlagen der additiven Fertigung kurz dargestellt. Wichtig für den weiteren Gang der Arbeit ist, dass mit einer Fertigungsdatei, die neben Informationen zur geometrischen Struktur des zu erzeugenden Objekts auch die zugehörigen Ausführungs- und Schichtinformationen umfasst, durch additive Fertigung auf einer geeigneten Fertigungsanlage ein komplexes Erzeugnis ohne weiteren Input eines Menschen erzeugt werden kann. Dies unterscheidet die additive Fertigung von anderen Herstellungsverfahren, bei denen Fachwissen notwendig ist, um einen Konstruktionsplan in ein körperliches Produkt umzusetzen.
$3 Z^{2 e c h}{ }^{1}$ folgend unterscheidet der Autor im informationstheoretischen Teil zwischen semantischer, struktureller und syntaktischer Information (Rz. 91 ff.). Semantische Information vermittelt Bedeutung. Sie stellt einen Bezug von Zeichen zu einem Sachverhalt ausserhalb ihrer selbst dar und kann Träger von Wahrheitswerten sein. Syntaktische Information betrifft die Menge von Zeichen und ihre Beziehungen zueinander. Strukturelle Information wohnt der räumlichen Anordnung von Elementen im Raum inne. Ihrer Natur nach wird Information nicht rivalisierend konsumiert, d.h. ihre Nutzung durch eine Person verhindert nicht, dass sie gleichzeitig einer beliebigen Vielzahl von weiteren Personen zur Verfügung steht. Dies soll gemäss dem Autor nicht für strukturelle Information gelten, was sich dem Rezensenten nicht erschliesst. Ohne entsprechende rechtliche Instrumente ist die Nutzung von Information zudem nicht ausschliessbar. Schliesslich wird Information bei der digitalen Übertragung notwendigerweise vervielfältigt, d.h. der Übermittler verbleibt in Besitz des Informationsträgers und damit der Information.

4 Im patentrechtlichen Grundlagenteil erläutert der Autor, dass das Patent seinem Inhaber Ausschliesslichkeitsrechte an der (gewerblichen) Nutzung einer technischen Handlungsanweisung (Lehre) verschafft (Rz. 137 ff.). Der schweizerische Gesetzestext nennt die Herstellung eines durch die technische Lehre beschriebenen Erzeugnisses dabei nur beispielhaft als eine Form der Nutzung (vgl. Art. 8 
Abs. 2 PatG²), während das deutsche Recht die dem Patentinhaber vorbehaltenen Nutzungshandlungen abschliessend aufzählt. Bildet ein Erzeugnis Gegenstand des Patents, so ist dessen Herstellung dem Patentinhaber vorbehalten (§ 9 Ziff. 1 DE-PatG). Nicht dem Patentinhaber vorbehalten ist das Zugänglichmachen der geschützten technischen Lehre, diese darf - anders als ein urheberrechtlich geschütztes Werk - frei mit Dritten geteilt werden. Die freie Verbreitung technischen Wissens rechtfertigt unter anderem die wettbewerbsbeschränkende Wirkung von Patenten.

5 Eine Herstellung liege vor, wenn eine Fachperson die Handlungsanweisungen der geschützten Lehre vollständig umsetze, wozu es bei traditioneller Herstellung neben der Information aus der Patentschrift des technischen Wissens der Fachperson bedürfe (vom Autor als «ausführende semantische Information» bezeichnet, in Abgrenzung zur «beanspruchenden semantischen Information» der geschützten Lehre, Rz. 172 ff.). Ein Erzeugnis entspreche daher der Verselbständigung der technischen Lehre, wobei «Verselbständigung» bedeute, dass die geschützte technische Lehre - nach ihrer Umsetzung in ein körperliches Erzeugnis - ohne weiteres Wissen über ihre Fertigung genutzt werden könne.

6 Nicht verlangt werde für die Herstellung ein körperliches Erzeugnis (Rz. 189 ff.). Das Erzeugnis mache die abstrakte technische Lehre verkehrsfähig und konsumierbar, dies könne auch auf andere Art geschehen als durch die Verkörperlichung der Lehre. Entscheidendes Ab-

Bundesgesetz vom 25. Juni 1954 über die Erfindungspatente (PatG; SR 232.14). grenzungskriterium sei nicht die Körperlichkeit, sondern die Art der Nutzung. Das Erzeugnis erlaube die Nutzung der beanspruchten technischen Lehre ohne weitere Informationen zu seiner Fertigung.

7 Ebenfalls nicht Voraussetzung eines Erzeugnisses sei die Fähigkeit, technische Wirkung(en) zu entfalten (Rz. 205 ff.). Dass ein körperliches Erzeugnis technische Wirkung entfalte, werde von der Rechtsprechung traditionellerweise verlangt, weil es ein Beweisanzeichen dafür sei, dass die semantische Information der geschützten Lehre vollständig umgesetzt worden sei. Wenn dieser Nachweis auf andere Art erbracht werde, könne auf das Erfordernis der technischen Wirkung verzichtet werden.

8 Nach dieser Vorarbeit ergibt sich die zentrale These ohne Weiteres (Rz. 213 ff.). Während bei der traditionellen Herstellung ausführendes Wissen der Fachperson und beanspruchte technische Lehre zu einem körperlichen Gegenstand führen, dessen strukturelle Information die gesamte semantische Information der Lehre spiegle, finde sich bei der additiven Fertigung die gesamte beanspruchte semantische Information in der Fertigungsdatei (dem syntaktischen Informationsträger). Entsprechend sei die Fertigungsdatei das Erzeugnis, und dessen Herstellung dem Patentinhaber vorbehalten. Das Erstellen einer Fertigungsdatei, die sämtliche Informationen zur körperlichen Herstellung eines Gegenstands enthält, der in den Schutzbereich des Erzeugnisanspruchs fällt, verletze das Patent unmittelbar (Rz. 220). Dies gelte auf jeden Fall dann, wenn das Material, aus dem der körperliche Gegenstand her- 
zustellen ist, im Anspruch nicht bestimmt werde (Rz. 216 ff.).

9 Wo der Patentanspruch die Verwendung eines bestimmten Materials oder eines Materials mit bestimmten Eigenschaften vorschreibe, sei mittels eines graduellen Tests zu entscheiden, ob das Zurverfügungstellen der Fertigungsdatei noch eine unmittelbare Patentverletzung darstelle (Rz. 232 ff.). In Anlehnung an BGH «Rigg»3 postuliert der Autor, auch wenn der Anspruch eine bestimmte Materialwahl verlange, könne im Zurverfügungstellen der Fertigungsdatei eine unmittelbare Patentverletzung gesehen werden, wenn die Datei ausschliesslich zur Fertigung patentgemässer körperlicher Erzeugnisse gebraucht werden könne und es zur Vollendung der Erfindung bloss der Hinzufügung selbstverständlicher, nebensächlicher Zutaten bedürfe (Rz. 284). Ob die Zutat (Materialwahl) untergeordnet ist, bestimme sich nach dem Aufwand für die Fertigung.

\section{Würdigung}

10 Die Dissertation von Vaterlaus-Abegg zeichnet sich durch die schlüssige Herleitung einer prägnanten These aus ihren Prämissen aus. Dadurch - und durch ihre erfreuliche Kürze von 121 Textseiten unterscheidet sie sich wohltuend von den leider allzu zahlreichen juristischen Dissertationen, die auf Hunderten von Seiten zusammentragen, was Rechtsprechung und Lehre zu einem Thema gesagt haben, ohne dass sich dem Leser erschliessen würde, was der Autor daraus ableitet.
11 Praktisch ist die Auffassung von Vaterlaus-Abegg in der Schweiz wichtig, weil das Schweizer Patentgesetz Teilnahmehandlungen an Patentverletzungen ausschliesslich akzessorisch konstruiert (vgl. Art. 66 lit. d PatG). Ein gewerblicher Anbieter von Fertigungsdateien für die additive Fertigung von patentgeschützten Erzeugnissen, der sein Angebot ausschliesslich an Private richtet, wirkt mangels einer Haupttat - die private Nutzung der geschützten Lehre ist erlaubt (Art. 9 Abs. 1 lit. a PatG) - nicht an einer Patentverletzung mit. 4 In Deutschland, dessen Patentrecht einen eigenständigen Tatbestand der mittelbaren Patentverletzung kennt ( $\S 10$ DE-PatG), kann das Zurverfügungstellen einer entsprechenden Fertigungsdatei auch dann verboten werden, wenn es als mittelbare Patentverletzung qualifiziert wird.

12 Im Übrigen hängt die praktische Bedeutung der Arbeit davon ab, ob die private Herstellung mit additiven Fertigungstechniken eine erhebliche wirtschaftliche Bedeutung erlangen wird. Das darf man bezweifeln. Das Verdienst, die Schutzwirkung des Patents informationstheoretisch analysiert zu haben, steht der Arbeit dennoch zu.

«Die Patentverletzung durch additive Fertigung (3D Druck)», Lukas AbeggVaterlaus, Carl Heymanns Verlag, Köln 2018, ISBN 978-3-452-29220-9 (gedruckte Ausgabe, Hardcover)
$3 \overline{\text { Urteil des BGH X ZR 70/80 vom 10. Dezember }}$ 1981 - «Rigg».
4 Str., siehe Andri Hess-Blumer/Roman Baechler, in: Schweizer/Zech (Hrsg.), SHK Patentgesetz, 2019, N 32 ff. zu Art. 66 PatG, m.H. 